\title{
Real-time Terrain Mode Recognition Module for the Control of Lower-limb Prosthesis
}

\author{
Yifei Pan ${ }^{1}$, Jiwen Zhang ${ }^{2}$, Libin Song ${ }^{3}$ and Chenglong $\mathrm{Fu}^{4, *}$ \\ ${ }^{123}$ State Key Laboratory of Tribology, Tsinghua University, Beijing, China, 100084 \\ ${ }^{4}$ Department of Mechanical and Energy Engineering, Southern University of Science and Technology, Shenzhen, China, 518055 \\ ${ }^{*}$ Corresponding author
}

\begin{abstract}
This paper is aimed to develop and evaluate a realtime terrain mode recognition system to assist the control of the lower-limb prosthesis. A wearable terrain recognition device, composed of an inertia measurement unit and a 2D-laser, was built to classify the types of terrain in front of the lower-limb prosthesis while walking. The terrain mode recognition module could not only detect the types of terrain in front of the wearers but also provide the detailed environment information such as the height of the stair, the angle of the slope. The built terrain mode recognition algorithm was evaluated on an able-bodied subject in the study. The results showed that the algorithm provided the terrain information accurately and quickly: terrain mode recognition accuracy was over $95 \%$ and the types of terrain were classified every $150 \mathrm{~ms}$ which was quick enough for the control of the lower-limb prosthesis in the future. Compared with the previous work on the terrain mode recognition, the approach mentioned on this paper could be applied in more complex environment and furthermore should be considered for operating wearable lower-limb prosthesis and orthotics.
\end{abstract}

Keywords - terrain recognition; 2D-laser; lower-limb prosthesis

\section{INTRODUCTION}

The previous research showed that powered lower-limb prosthesis is able to improve the athletic performance for those tibial amputations. [1,2] Much research in recent years has focused on how to control the motion of powered lower-limb prosthesis to assist amputations walking on the different terrains [3-5]. However, the movement tasks in daily living are more complex. Thus it is essential to switch the control mode timely and quickly when walking on the different terrains. Because the basis on which prosthesis can switch the control mode is obtaining the change of terrain ahead of the amputations, it is important to purpose the methods to obtain the environment information in front of the amputations.

For some commercially available lower-limb exoskeletons and prosthesis, users can simply "tells" the prosthesis an intended joint motion or execute a certain movement by pushing the control bottoms, as is used in ReWalk system (Argo Medical Technologies Ltd. Israel), or by given body gestures, as employed in the Power Knee (Iceland). However, it is unfriendly for amputations to remember the complex certain body motions. Additionally, amputations have to stop walking, finish the certain body motions and then go upstairs which makes motion unconscious.
Hence, some groups obtained the environment information via the inertia measurement unit fixed on the powered ankle prosthesis to determine the terrain ahead of the amputations [6]. The results showed that wears can walk on the ground, go up ramp, go upstairs and downstairs consciously and quickly. However, the IMU could not obtain the change of terrain unless the amputations walk on the new terrain, thus the time for the prosthesis to switch the control mode is limited. Some groups purposed an approach based on the IMU and a laser distance meter which can obtain the change of terrain's height ahead of the amputations and classify the types of terrain. [7]

However, the approaches above still have some disadvantages. Since the laser distance meter sensor can just obtain the data point about one or two steps ahead, there is a blind area within one or two steps so that the methods couldn't recognize the change of terrain close to the wear especially when the wears walk on the staircase. Second, the types of the terrain are simply classified by the difference in height between the waist and the reflection point which may led to mistakes up ramp from up stair.

The purpose of this study is to develop and evaluate a realtime terrain recognition system to assist the control of the powered lower-limb prosthesis. This paper is organized as follows:1) the design of a new terrain mode recognition device to obtain the $2 \mathrm{D}$ environment information (on the sagittal plane) ahead of the prosthesis. 2) the design of a new terrain mode recognition algorithm based on the environment information to classify the terrain mode. 3) a preliminary experiment to evaluate the performance of the algorithm.

\section{METHOD}

In this section, we focused on the design of the real-time terrain mode recognition device and developing the algorithm used for the terrain mode recognition.

\section{A. Design of the Terrain Mode Recognition Device}

Although many terrain recognition systems have been applied in autonomous land vehicle systems and technology based on 2-D lasers combined with a camera, and 3-D laser scanners [8], these are hardly used for the control of the lowerlimb prosthesis and the systems are little complex as wearable devices for prosthesis. In this study, a lightweight wearable terrain mode recognition device was designed to recognize the terrain ahead of the lower-limb prosthesis wear. The system 
consist of an inertia measurement unit (IMU) , a 2-D lasers and a Raspberry Pi.

The placement of the sensors is shown on Figure I. The IMU was fixed on the shell of the 2-D laser in order to get the attitude of the laser. The IMU measured the acceleration and angular velocity of the laser and thigh. The laser measured the distance between the center of sensor and the laser reflection point on the terrain and the angle from vertical direction to reflection ray[d and $\alpha$ in Figure I.]. In this study, the sensors were placed on the thigh fixed on the lower-limb prosthesis in order to make the sensors be a part of the prosthesis and thus be easier to wear for the prothesis wears.

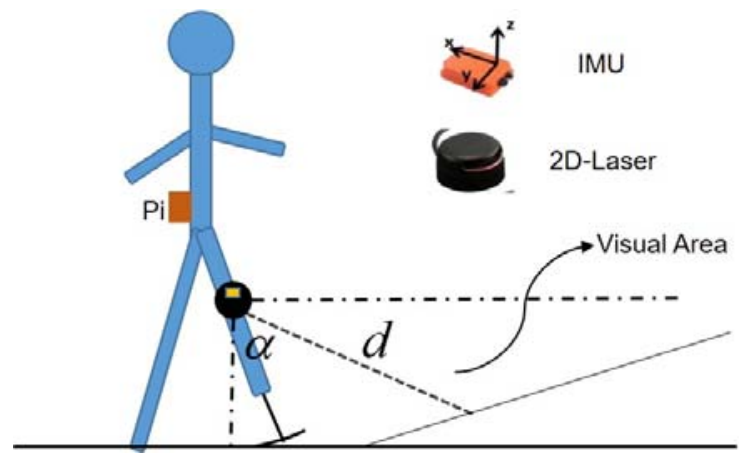

FIGURE I. THE PLACEMENT OF THE SENSORS

The Raspberry Pi placed on the waist is used to analysis the data and communicate with the lower-limb prosthesis via the RS-232.

\section{B. Design of the Terrain Mode Recognition Algorithm}

The terrain mode recognition algorithm contains two basic function module: terrain mode classification module and terrain information calculation module. The measurements of the IMU were used to obtain the pose of the 2-D laser and the measurements of the laser were used to recognize the terrain ahead of the lower-limb prosthesis.

\section{1) Terrain mode classification module:}

The geometry data obtained from the 2D-laser sensor is the distance between the sensor center and the reflection point on the ground and the angle between the zero line and the laser reflection ray in the sensor coordinate system. When wears walk on the ground, the visual range we are concerned about is from the vertical direction to the horizontal direction, so the data out of this range is unnecessary. Since the zero-line changes with the movement of wears, an IMU is employed to measure the sensor pose: the Y-axis of the IMU is fixed with the zero line of the laser sensor when the wearer is standing still and upright, then the angle of Y-axis rotation is the same as the angle of zero-line rotation. Thus, the data obtained is always from the vertical direction to the horizontal direction on the sagittal plane.

The original data obtained from the laser sensor is based on the sensor polar coordinate system, in order to recognize the terrain, the data is translated to the cartesian coordinate system s. The transition matrix is as follow:

$$
\left[\begin{array}{l}
x[n] \\
y[n]
\end{array}\right]=\left[\begin{array}{l}
\sin \alpha[n] \\
\cos \alpha[n]
\end{array}\right] d[n]
$$

Where $d[n]$ is the $n$th reading of the laser reflection point and $\alpha[\mathrm{n}]$ denotes the angle on the polar coordinate system.

The data on the cartesian coordinate system is discrete. In order to classify the types of terrain, Douglas-Peucker algorithm, a kind of vector compression algorithm, is applied to segment the data, the segmentation points are then used for the classification.

The terrain classification was realized by a three-layer decision tree (Figure II). The first layer of the decision tree classified the terrain into two different nodes: level ground(LG) and non-level ground(NLG). If the recognition result is NLG, the second decision layer of the decision tree was then employed. The second decision layer further classified NLG into four different nodes: stair(STA), ramp(RA), step(STE) and others. The third decision layer of the decision tree further classified STA into two different nodes: up stair(US) and down stair(DS), classified RA into two different nodes: up ramp(UR) and down $\operatorname{ramp}(\mathrm{DR})$.

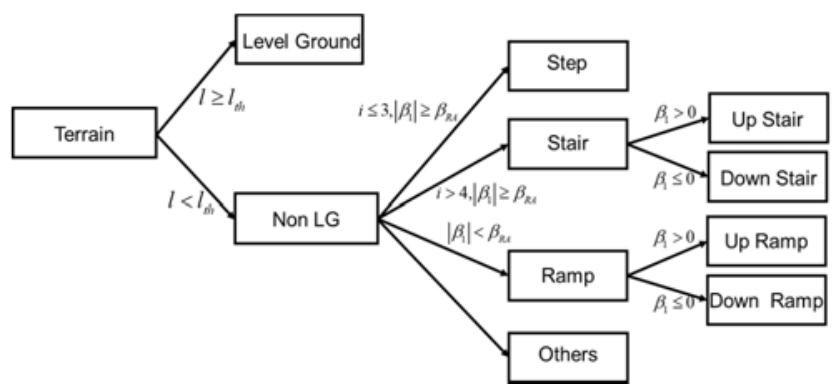

FIGURE II. THE DECISION TREE FOR RECOGNITION

The classification result of the first layer was based on the assumption that there was at least a bit of level ground ahead of the wears. The length of the level ground was calculated by:

$$
l=\sqrt{\left(X_{2}-X_{1}\right)^{2}+\left(Y_{2}-Y_{1}\right)^{2}}
$$

Where $l$ is the length of the level ground and $\left(X_{1}, Y_{1}\right),\left(X_{2}, Y_{2}\right)$ denotes the first and second segment points, shown on the Figure III. 


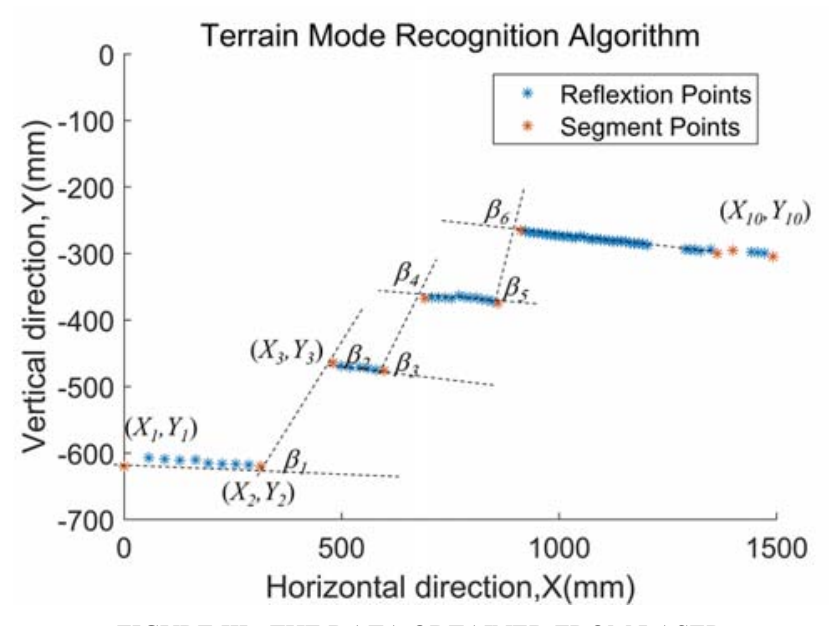

FIGURE III. THE DATA OBTAINED FROM LASER

The first layer of the decision tree identified terrains by comparing the measured length of the level ground $l$ with predefined thresholds. If the $l$ is more than $l_{\text {th }}$, which was set to $750 \mathrm{~mm}$ (the average step length of an adult) then the recognition result was LG. Otherwise, the recognition result was NLG.

It was obvious that if the decision was NLG, there was more then three segment points obtained from the laser sensor. The slope $\mathrm{k}_{\mathrm{i}}$ between $\left(X_{i+1}, Y_{i+1}\right)$ and $\left(X_{i}, Y_{i}\right)$ was calculated as follow:

$$
k_{i}=\frac{Y_{i+1}-Y_{i}}{X_{i+1}-X_{i}}
$$

Then the slope change of different segment line was defined as:

$$
\beta_{\mathrm{i}}=\arctan k_{\mathrm{i}+1}-\arctan k_{i}
$$

The thresholds $\beta_{R A}$, was set as the standard to distinguish the RA and the other terrains. If $\left|\beta_{1}\right| \leq \beta_{R A}$, then the classification result was RA. What's more, if $\beta_{1}>0$, the classification result of the third layer was UR, otherwise, the classification result of the third layer was DR.

$$
\text { If }\left|\beta_{1}\right| \geq \beta_{R A} \text {, then numbers of }\left|\beta_{\mathrm{i}}\right| \geq \beta_{R A} \text { were calculated. }
$$

If $i \leq 3$ then the classification was STE, otherwise the decision was STA. If the decision was STA, then if $\beta_{1}>0$, the classification result of the third layer was US and else the result was DS.

However, there were other terrains like obstacles, walls, chairs, they were defined as the others in the study.

\section{2) Terrain mode classification module:}

Besides the classification of the types of the terrain, the terrain recognition algorithm can also calculate the detail information. The length of level ground is calculated by the distance between the first and second segment points. The angle of the ramp is the length of the ramp is the distance between the second and third segment points. The number of stair steps is calculated as follow:

$$
N=0.5 i
$$

At the same time, the height and length of the stair is calculated by:

$$
\begin{aligned}
& H_{\text {stair }}=\frac{1}{N} \sum_{i=1}^{N}\left|Y_{2 i+1}-Y_{2 i}\right| \\
& L_{\text {stair }}=\frac{1}{N} \sum_{i=1}^{N}\left|X_{2 i+2}-X_{2 i+1}\right|
\end{aligned}
$$

Compared with the previous work, the algorithm applied in the study could not only classify the types of terrain in front of the wears but also output the detail environment information which could be future used in the control of the powered lowerlimb prosthesis.

\section{EXPERIMENT AND RESULTS}

The performance of the terrain mode recognition module was evaluated on an abled-body subject on line (shown on the Figure IV). The evaluation experiments contained two different parts:

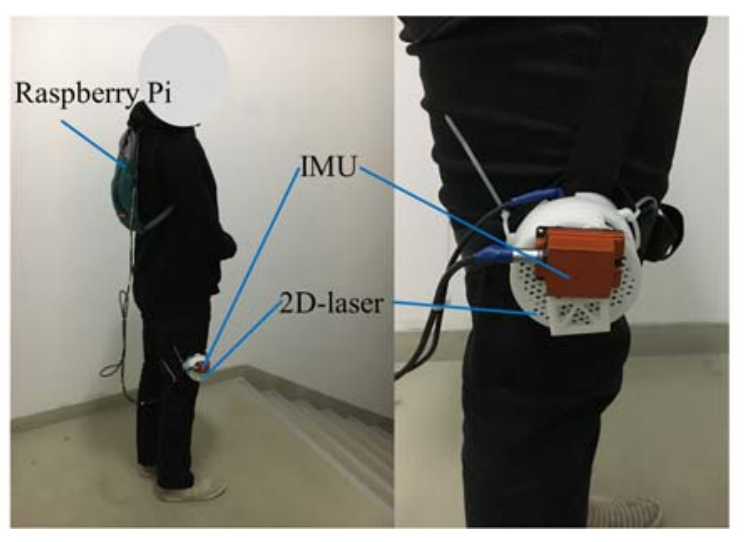

FIGURE IV. ABLED-BODY SUBJECT WEARING DEVICE

The first experiment was used to evaluate the accuracy of the terrain mode recognition module. The classification accuracy is defined as the percentage of correct terrain recognition results out of the total terrain recognition results. The results were shown on Table I. 
TABLE I. THE ACCURACY OF THE MODULE

\begin{tabular}{|c|c|c|c|}
\hline \multirow{2}{*}{$\begin{array}{c}\text { The mode of the } \\
\text { terrain }\end{array}$} & \multicolumn{3}{|c|}{ The result of the experiment } \\
\cline { 2 - 4 } & $\begin{array}{c}\text { Correct } \\
\text { Number }\end{array}$ & $\begin{array}{c}\text { Total } \\
\text { Number }\end{array}$ & ACCURACY \\
\hline LG & 1233 & 1252 & $98.48 \%$ \\
\hline US & 1921 & 2006 & $95.76 \%$ \\
\hline DS & 1784 & 1872 & $95.29 \%$ \\
\hline UR & 1925 & 1985 & $96.97 \%$ \\
\hline DR & 1527 & 1583 & $96.46 \%$ \\
\hline STE & 1285 & 1324 & $97.05 \%$ \\
\hline
\end{tabular}

The results showed that the accuracy of the terrain mode recognition module was over $95 \%$. However, there were still some error decisions given by the module, which indicated that the decision tree could be improved in the future work.

The second experiment was used to evaluate the performance of the module while walking on the staircase. The results were shown on Figure V, where the "W" means walking on the ground, "SA" means walking upstairs and "SD" means walking downstairs. The results showed that the method could provide the terrain information every $150 \mathrm{~ms}$ and when the subject walked from level ground to the stair, there were always an error classification result "STEP". That was because the last step walking upstairs or downstairs was actually a stair which the number of steps was 1 however in our decision tree that was regarded as the "STEP".

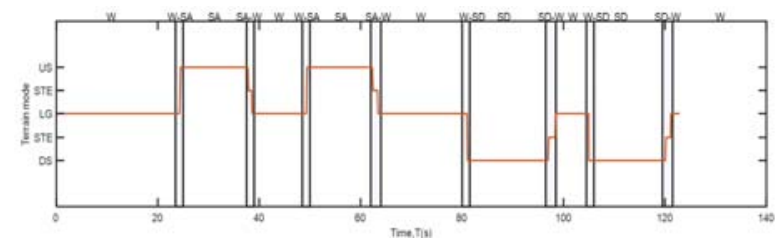

FIGURE V. THE DATA OBTAINED FROM LASER

The height and length of the stair was also calculated on this experiment. The height of the stair was $150 \mathrm{~mm}$ and the length of the stair was $280 \mathrm{~mm}$ in our study. The height of stair calculated by the module was $151.52 \mathrm{~mm} \pm 2.32 \mathrm{~mm}$. The length of stair calculated by the module was $332.27 \mathrm{~mm} \pm 25.17 \mathrm{~mm}$. The result showed that the performance on the height direction was better than the performance on the length direction, that may because when subject walked on the staircase, the walking direction was not parallel to the length direction, which led to the calculated result was more than the actual length of the stair. And an example of the module output was shown on Figure VI.

\section{CONCLUSION}

In this paper, a real-time terrain recognition system was developed and evaluated online. A terrain mode recognition algorithm based on a decision tree was designed to classify the types of terrain in front of the lower-limb prosthesis. The experiments showed that the algorithm given the real-time terrain information per $150 \mathrm{~ms}$ which was quick enough for prothesis to translate the control mode. The methods performed well during walking on the staircase. The developed device showed the potentiality to assist the control of the lower-limb prosthesis. Meanwhile, the information produced by the terrain recognition algorithm was not only useful to assist the control of the lower-limb prosthesis but also for the control of the other lower-limb wearable robotics.

\section{ACKNOWLEDGMENT}

This research was supported in part by National Natural Science Foundation of China under Grant 61533004, Grant U1613206, and in part by Beijing Municipal Science \& Technology Commission (No Z161100000516033).

\section{REFERENCES}

[1] Grabowski, Alena M, and Susan D'Andrea, "Effects of a powered anklefoot prosthesis on kinetic loading of the unaffected leg during levelground walking".Journal of neuroengineering and rehabilitation, 10(1), 2013, pp. 49.

[2] Au, Samuel K., Jeff Weber, and Hugh Herr, "Powered Ankle--Foot Prosthesis Improves Walking Metabolic Economy". IEEE Transactions on Robotics, 25(1), 2009, pp. 51-66.

[3] Sup, Frank, Amit Bohara, and Michael Goldfarb, "Design and control of a powered transfemoral prosthesis". The International journal of robotics research 27(2), 2008, pp. 263-273.

[4] Eilenberg, Michael F., Hartmut Geyer, and Hugh Herr, "Control of a powered ankle-foot prosthesis based on a neuromuscular model". IEEE transactions on neural systems and reh abilitation engineering 18(2), 2010, pp. 164-173.

[5] Au, Samuel, Max Berniker, and Hugh Herr, "Powered ankle-foot prosthesis to assist level-ground and stair-descent gaits". Neural Networks 21(4), 2008, pp.654-666.

[6] Wang, Q., Yuan, K., Zhu, J., \& Wang, L, "Walk the walk: A lightweight active transtibial prosthesis". IEEE Robotics \& Automation Magazine 22(4), 2015, pp. 80-89.

[7] Liu, Ming, Ding Wang, and He Helen Huang, "Development of an environment-aware locomotion mode recognition system for powered lower limb prostheses". IEEE Transactions on Neural Systems and Rehabilitation Engineering 24(4), 2016, pp. 434-443.

[8] Durrant-Whyte, Hugh, and Tim Bailey, "Simultaneous localization and mapping: part I". IEEE robotics \& automation magazine 13(2), 2006, pp. 99-110.

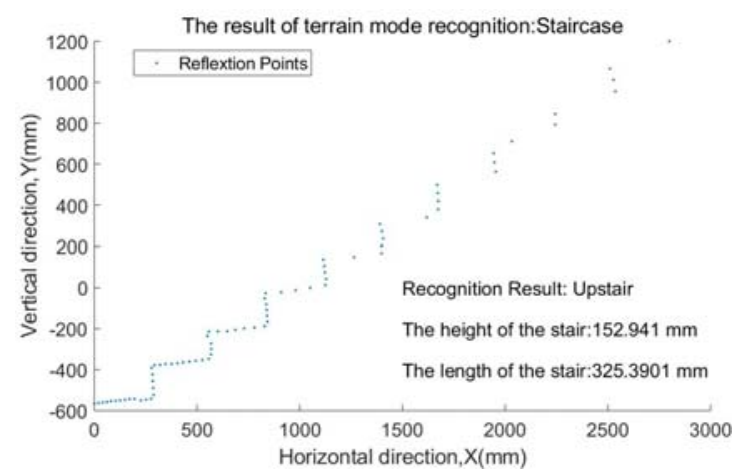

FIGURE VI. THE RESULT OF TERRAIN MODE RECOGNITION :STAIRCASE 